\title{
Flutter Suppression of Long-Span Bridges Using Suboptimal Control
}

\author{
Lingjun Zhuo', Yunjin Dong'2, Xinyu $\mathrm{Xu}^{3}$ \\ ${ }^{1}$ Research Center for Wind Engineering, Southwest Jiaotong University, Chengdu, China \\ ${ }^{2}$ Xihua University, Chengdu, China \\ ${ }^{3}$ China Railway Eryuan Engineering Group Co., Ltd., Chengdu, China \\ Email:1jzhuo@126.com
}

How to cite this paper: Zhuo, L.J., Dong, Y.J. and Xu, X.Y. (2018) Flutter Suppression of Long-Span Bridges Using Suboptimal Control. World Journal of Engineering and Technology, 6, 34-40. https://doi.org/10.4236/wjet.2018.62B004

Received: March 13, 2018

Accepted: May 19, 2018

Published: May 22, 2018

\begin{abstract}
Based on the Theodorsen's Theory of the aerodynamic forces on wing-aileron, the Scanlan's Theory is expanded considering a deck-flap system. It is suggested that a new forced vibration method can acquire aerodynamic derivatives of this deck-flap system theoretically. After obtaining the wind induced forces, a deck-flap equation of motion in time domain is established to investigate its control law. Numerical simulation results indicate suboptimal control law of the deck-flap system can suppress the flutter effectively, and the flutter speed can be increased for desirable purpose.
\end{abstract}

\section{Keywords}

Aerodynamic Force, Deck-Flap System, Flutter Suppression, Control Law

\section{Introduction}

Flutter is a self-excited motion, which eventually leads to catastrophic damage in bridge structures. Nowadays, with more and more long-span bridges to emerge, finding ways to suppress flutter and increase stability can tackle severe wind-induced problems. Adding stiffness of a girder, application of mechanical dampers are common ways to improve a bridge aerodynamic property. Using active control is a new way to solve these problems.

Some researchers tried to put flaps away from deck in order to omit interference of aerodynamic forces between deck and flaps and can easily apply Theodorsen's Theory of aerodynamic forces [1]. However, this interference cannot be omitted and can improve aerodynamic property in a way [2] [3].

In this paper, the active control system composes of a deck and flaps symmetrically mounted adjacent to the deck. A deck-flap equation of motion in time 
domain is established. Along with a new forced vibration method, aerodynamic forces can be calculated theoretically. In the end, a numerical simulation helps to investigate its control law.

\section{Equation of Motion in Time Domain}

Flutter analysis is usually done in the frequency domain. The frequency-dependent motion-induced forces should be transformed to time-dependent ones so that they can be applied in the active control analysis. Based on the Scanlan's Theory, the two dimensional equation of motion can be expressed as:

$$
\begin{gathered}
m \ddot{h}+c_{h} \dot{h}+k_{h} h=L=L_{d}+L_{f} \\
I \ddot{\alpha}+c_{\alpha} \dot{\alpha}+k_{\alpha} \alpha=M=M_{d}+M_{f}
\end{gathered}
$$

where $m=$ mass of the system, $I=$ moment of inertia of the system, $c_{h}, c_{\alpha}=$ damping of vertical and torsional motion respectively, $k_{h}, k_{\alpha}=$ stiffness of vertical and torsional motion respectively, $L, M=$ total lift and moment respectively, $L_{d}, M_{d}=$ motion-induced lift and moment of the deck respectively, $L_{f}, M_{f}=$ motion-induced lift and moment of the flaps respectively.

The motion-induced forces of the deck can be expressed as:

$$
\begin{aligned}
& L_{d}=\frac{1}{2} \rho U^{2}(2 B)\left(K_{h} H_{1}^{*} \frac{\dot{h}}{U}+K_{h} H_{2}^{*} \frac{B \dot{\alpha}}{U}+K_{h}^{2} H_{3}^{*} \alpha+K_{h}^{2} H_{4}^{*} \frac{h}{B}\right) \\
& M_{d}=\frac{1}{2} \rho U^{2}\left(2 B^{2}\right)\left(K_{\alpha} A_{1}^{*} \frac{\dot{h}}{U}+K_{\alpha} A_{2}^{*} \frac{B \dot{\alpha}}{U}+K_{\alpha}^{2} A_{3}^{*} \alpha+K_{\alpha}^{2} A_{4}^{*} \frac{h}{B}\right)
\end{aligned}
$$

where $\rho=$ air density, $U=$ wind velocity, $B=$ deck width, $h, \alpha=$ vertical and torsional displacement respectively, $K_{h}=\omega_{h} B / U, K_{\alpha}=\omega_{\alpha} B / U$, $H_{i}^{*}, A_{i}^{*}(i=1 \sim 4)=$ aerodynamic derivatives of the deck.

The aerodynamic flaps can be driven on both sides. Figure 1 shows that when flutter of the system is detected, the trailing flap is motivated and the leading flap is locked along with the deck. In this way, Theodorsen's Theory on wing-aileron forces can be applied [4].

$$
\begin{aligned}
L_{f} & =\frac{1}{2} \rho U^{2}(2 B)\left(K_{h} H_{5}^{*} \frac{B \dot{\beta}}{U}+K_{h}^{2} H_{6}^{*} \beta\right) \\
M_{f} & =\frac{1}{2} \rho U^{2}\left(2 B^{2}\right)\left(K_{\alpha} A_{5}^{*} \frac{B \dot{\beta}}{U}+K_{\alpha}^{2} A_{6}^{*} \beta\right)
\end{aligned}
$$

where $\beta=$ torsional displacement of trailing flap, $H_{i}^{*}, A_{i}^{*}(i=5,6)=$ aerodynamic derivatives of the trailing flap.

To obtain the aerodynamic derivatives for the deck-flap system, a forced

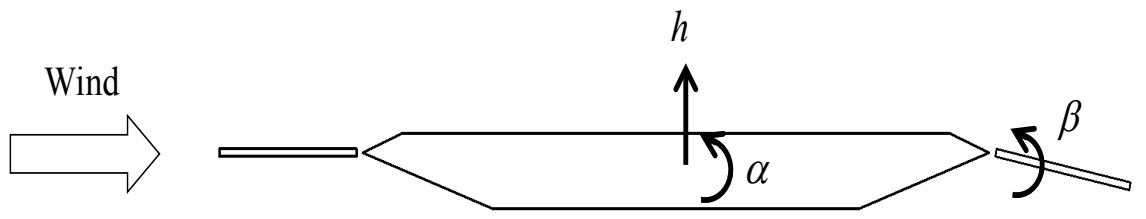

Figure 1. Deck-flap system [2]. 
vibration method is proposed. When the system is forced to rotate sinusoidally, displacements of the system can be assumed as:

$$
h=0 \quad \alpha=\alpha_{0} e^{i \omega t} \quad \beta=\beta_{0} e^{i(\omega t+\varphi)}
$$

The total lift and moment are:

$$
\begin{aligned}
L & =\frac{1}{2} \rho U^{2}(2 B)\left[K\left(H_{2}^{*}+l_{1} H_{5}^{*}+l_{2} H_{6}^{*}\right) \frac{B \dot{\alpha}}{U}+K^{2}\left(H_{3}^{*}+l_{3} H_{5}^{*}+l_{4} H_{6}^{*}\right) \alpha\right] \\
& =\frac{1}{2} \rho U^{2}(2 B)\left(K \hat{H}_{2} \frac{B \dot{\alpha}}{U}+K^{2} \hat{H}_{3} \alpha\right) \\
M & =\frac{1}{2} \rho U^{2}\left(2 B^{2}\right)\left[K\left(A_{2}^{*}+m_{1} A_{5}^{*}+m_{2} A_{6}^{*}\right) \frac{B \dot{\alpha}}{U}+K^{2}\left(A_{3}^{*}+m_{3} A_{5}^{*}+m_{4} A_{6}^{*}\right) \alpha\right] \\
& =\frac{1}{2} \rho U^{2}\left(2 B^{2}\right)\left(K \hat{A}_{2} \frac{B \dot{\alpha}}{U}+K^{2} \hat{A}_{3} \alpha\right)
\end{aligned}
$$

where $l_{i}, m_{i}(i=1,2,3,4)=$ a combination of flap's amplitude and its phase angle, $\hat{H}_{i}, \hat{A}_{i}(i=2,3)=$ new derivatives of the system in the forced vibration method.

Using the same way in the regular forced vibration method, the new derivatives can be acquired from:

$$
\begin{array}{ll}
\hat{H}_{2}=\frac{2}{\rho B^{3} \omega^{2} \alpha_{0}} \operatorname{Im}[F(L)] & \hat{H}_{3}=\frac{2}{\rho B^{3} \omega^{2} \alpha_{0}} \operatorname{Re}[F(L)] \\
\hat{A}_{2}=\frac{2}{\rho B^{4} \omega^{2} \alpha_{0}} \operatorname{Im}[F(M)] & \hat{A}_{3}=\frac{2}{\rho B^{4} \omega^{2} \alpha_{0}} \operatorname{Re}[F(M)]
\end{array}
$$

And their relations can be put as:

$$
\begin{aligned}
& {\left[\begin{array}{c}
\hat{H}_{2} \\
\hat{H}_{3}
\end{array}\right]=\left[\begin{array}{llll}
1 & 0 & l_{1} & l_{2} \\
0 & 1 & l_{3} & l_{4}
\end{array}\right]\left[\begin{array}{llll}
H_{2} & H_{3} & H_{5} & H_{6}
\end{array}\right]^{T}} \\
& {\left[\begin{array}{c}
\hat{A}_{2} \\
\hat{A}_{3}
\end{array}\right]=\left[\begin{array}{llll}
1 & 0 & m_{1} & m_{2} \\
0 & 1 & m_{3} & m_{4}
\end{array}\right]\left[\begin{array}{llll}
A_{2} & A_{3} & A_{5} & A_{6}
\end{array}\right]^{T}}
\end{aligned}
$$

As seen from above, it needs only a few tests to figure out a statically indeterminate matrix for solving the aerodynamic derivatives. Thus, inserting Equations (2) and (3) into Equation (1), and transforming it into Laplace domain with zero initial conditions gives:

$$
\left[\bar{M} s^{2}+\bar{C} s+\bar{K}\right] \tilde{q}=\tilde{F}
$$

where

$$
\bar{M}=\left[\begin{array}{cc}
m B & \\
& I
\end{array}\right], \bar{C}=\left[\begin{array}{ll}
c_{h} B & \\
& c_{\alpha}
\end{array}\right], \quad \bar{K}=\left[\begin{array}{cc}
k_{h} B & \\
& k_{\alpha}
\end{array}\right], \quad \tilde{q}=\left[\begin{array}{c}
\tilde{h} / B \\
\tilde{\alpha}
\end{array}\right], \quad \tilde{F}=\left[\begin{array}{c}
\tilde{Q}_{L} \\
\tilde{Q}_{M}
\end{array}\right]
$$

A common way to transform wind induced forces into time domain is a rational function approximation by Roger [5]. The details can be found in any aeronautical textbook. Each coefficient $Q_{i j}$ can be expressed as:

$$
Q_{i j}=A_{0}^{i j}+A_{1}^{i j} \bar{s}+\sum_{m=2}^{N} A_{m}^{i j} \frac{\bar{s}}{\bar{s}+\gamma_{m-1}}
$$


Combined with aerodynamic derivatives acquired from forced vibration method, each coefficient can be obtained through the rational function approximation.

\section{Feedback Control}

Equation of motion can be rewritten as:

$$
\bar{M} \ddot{q}+\bar{C} \dot{q}+\bar{K} q-q_{d}\left(A_{0}+A_{1} \bar{s}+\sum_{m=2}^{N} A_{m} \frac{\bar{s}}{\bar{s}+\gamma_{m-1}}\right) q=q_{d}\left(B_{0}+B_{1} \bar{s}+\sum_{m=2}^{N} B_{m} \frac{\bar{s}}{\bar{s}+\gamma_{m-1}}\right) \beta
$$

In order to study control law for stabilization, the equation of motion can be put in the state space form:

$$
\left\{\begin{array}{c}
\dot{x}=A x+B u \\
y=C x
\end{array}\right.
$$

where,

$$
\begin{aligned}
& x=\left[\begin{array}{c}
X \\
\dot{X} \\
X_{a 3} \\
\vdots \\
X_{a m}
\end{array}\right], A=\left[\begin{array}{ccccc}
0 & I & 0 & \cdots & 0 \\
-M^{-1} K & -M^{-1} C & q_{d} M^{-1} A_{3} & \cdots & q_{d} M^{-1} A_{m} \\
0 & I & -\frac{U \gamma_{1}}{B} I & \cdots & 0 \\
\vdots & \vdots & \vdots & \ddots & \vdots \\
0 & I & 0 & \cdots & -\frac{U \gamma_{m-2}}{B} I
\end{array}\right] \text {, } \\
& B=\left[\begin{array}{c}
0 \\
M^{-1} G \\
0 \\
\vdots \\
0
\end{array}\right], u=\beta_{c}, y=[X], \quad C=\left[\begin{array}{cccc}
I & & & \\
& 0 & & \\
& & \ddots & \\
& & & 0
\end{array}\right] \text {, } \\
& M=\bar{M}-q_{d} \frac{B^{2}}{U^{2}} A_{2}, \quad C=\bar{C}-q_{d} \frac{B}{U} A_{1}, \quad K=\bar{K}-q_{d} A_{0}, \quad G=\left[\begin{array}{c}
0 \\
0 \\
k_{c} k_{\beta}
\end{array}\right] \text {. }
\end{aligned}
$$

Optimal output control is a mature way [3] [6], but it needs all the state vector to be measured, which isn't easy in the deck-flap system. On the other hand, suboptimal output control can be exerted through only a few state vector. It's more reliable in wind tunnel experiments [7]. Suppose that the control is generated via output linear feedback gains:

$$
u=-K_{c o n} y
$$

where $K_{c o n}=$ feedback gain matrix to be determined.

To solve the suboptimal output control problem is to figure out a optimization of the averaged performance index:

$$
J=\frac{1}{2} \int_{0}^{\infty}\left(x^{T} Q x+u^{T} R u\right) d t
$$

where $Q, R=$ appropriate weighting matrices. 
Inserting the output equation from the state space form and performing a simple mathematical operation yields

$$
\begin{aligned}
& Q J=\frac{1}{2} \int_{0}^{\infty}\left(x^{T} Q x+x^{T} C^{T} K_{c o n}^{T} R K_{c o n} C x\right) d t \\
& =\frac{1}{2} \int_{0}^{\infty} x^{T}\left(Q+C^{T} K_{c o n}^{T} R K_{c o n} C\right) x d t=\frac{1}{2} \int_{0}^{\infty} x^{T} Q_{1} x d t
\end{aligned}
$$

It appears that $K_{c o n}$ is the solution of three equations:

$$
\begin{gathered}
\min J=\min \frac{1}{2} \int_{0}^{\infty} x^{T} Q_{1} x d t \\
\dot{x}=A_{c o n} x=\left(A-B K_{c o n} C\right) x \\
P A_{c o n}+A_{c o n}^{T} P+Q_{1}=0
\end{gathered}
$$

\section{Numerical Simulation}

A plate with decks on both sides is simulated to see the results of the application of active control. The deck is 40 meters wide, mass of the deck $m=20000 \mathrm{~kg} / \mathrm{m}$, moment of inertia $I=4.5 \times 10^{6} \mathrm{~kg} \cdot \mathrm{m}^{2} / \mathrm{m}, \omega_{h}=0.1788 \mathrm{~Hz}$, $\omega_{\alpha}=0.5028 \mathrm{~Hz}$, air density $\rho=1.225 \mathrm{~kg} / \mathrm{m}^{3}$, the width of flap is $3 \mathrm{~m}$.

First, its aerodynamic forces should be transformed through the Roger approach, as shown in Figure 2.

The aim of the active control is to suppress flutter up to $159 \mathrm{~m} / \mathrm{s}$. when using optimal control, the value of its gain is:

$$
\begin{aligned}
& K_{o p}=\left[\begin{array}{lllllll}
-0.5373 & 2.8299 & 3.6154 & -1.8600 & 0.0069 & 1.0017
\end{array}\right. \\
& \begin{array}{llllll}
2.5807 & -6.5471 & -1.6502 & 3.5028 & -1.0229 & -4.3174]
\end{array}
\end{aligned}
$$

Figure 3 shows the responses of the deck-flap system under optimal control in the wind speed of $159 \mathrm{~m} / \mathrm{s}$, when the system is exerted external forces.

The value of gain after applying suboptimal control is:

$$
K_{\text {con }}=\left[\begin{array}{ll}
-0.0098 & 0.6779
\end{array}\right]
$$

And its responses in the wind speed of $159 \mathrm{~m} / \mathrm{s}$ is shown in Figure 4, when exerted with external forces.

The numerical example shows that both control laws can suppress flutter in the high wind speed. Despite the quick response under optimal control, it's hard to track all the parameters in state vector. The suboptimal control provides a brief strategy to suppress flutter with few parameters in state vector. In this case, the control law depends on the vertical and torsional displacements. For wind tunnel experiments, the two displacements are very common and easy to track.

\section{Conclusions}

The modeling of deck-flap system is studied in this paper. A state space form of equations of motion in time domain are obtained through Roger's approach.

Considering the interference between the deck and flaps, a forced vibration method is proposed. Theoretically, this method can help researchers obtain 

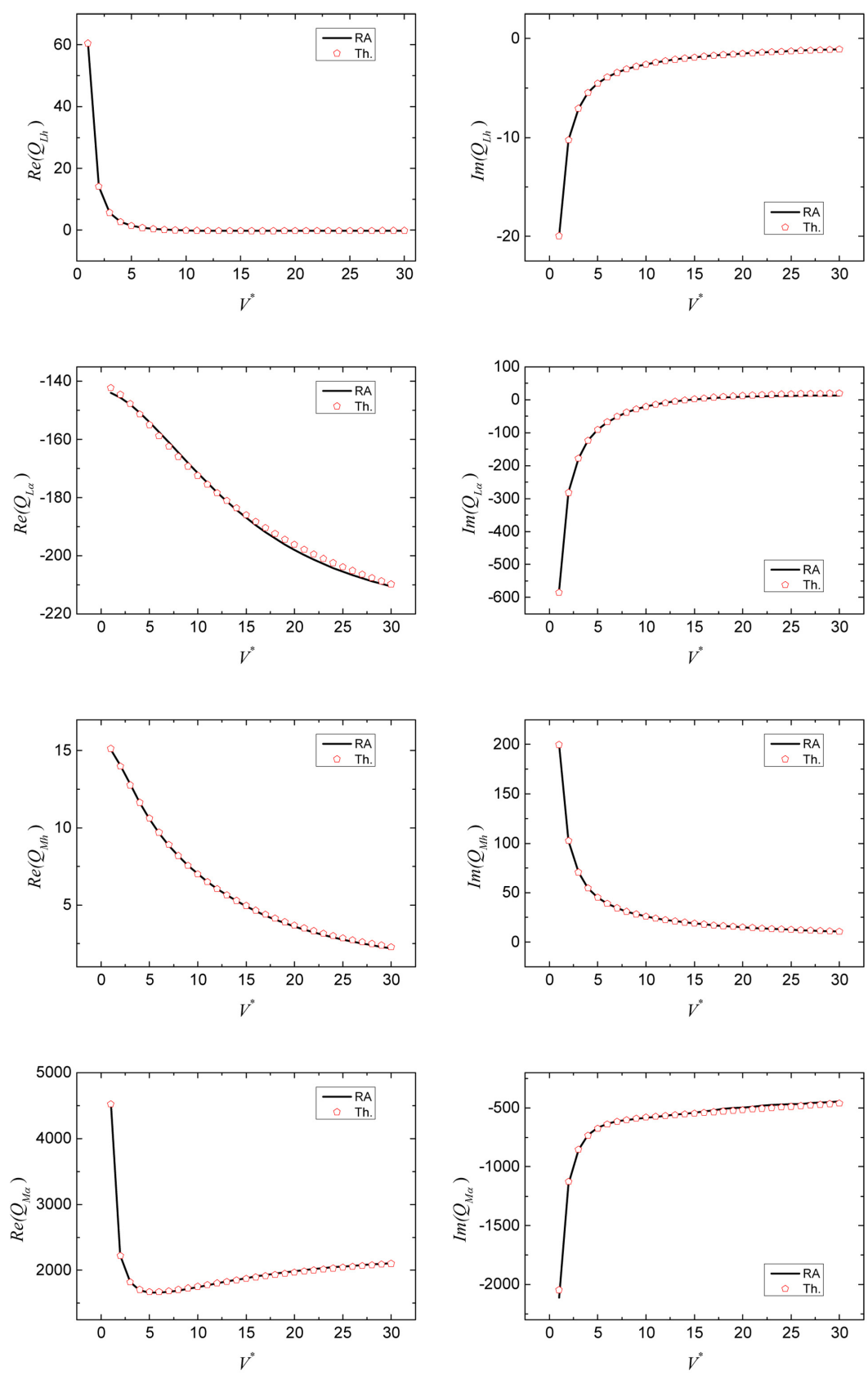

Figure 2. Rational approximation for motion-induced forces.
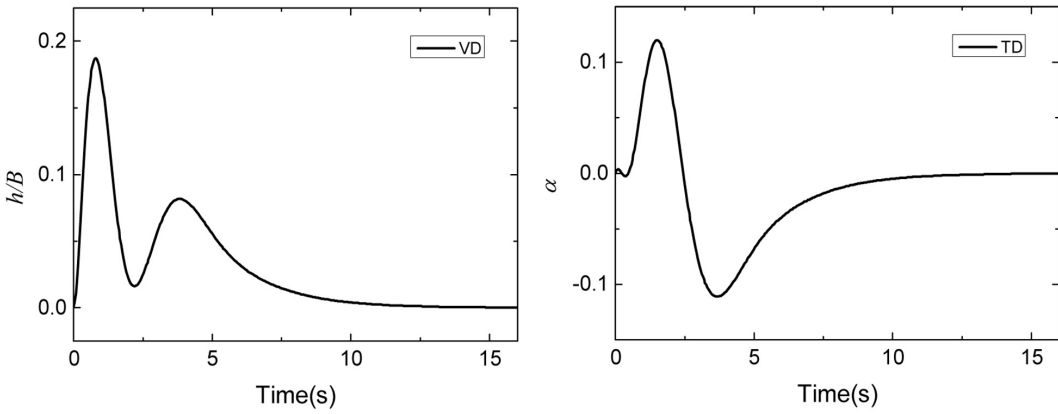

Figure 3. Time-history of system displacements under optimal control. 

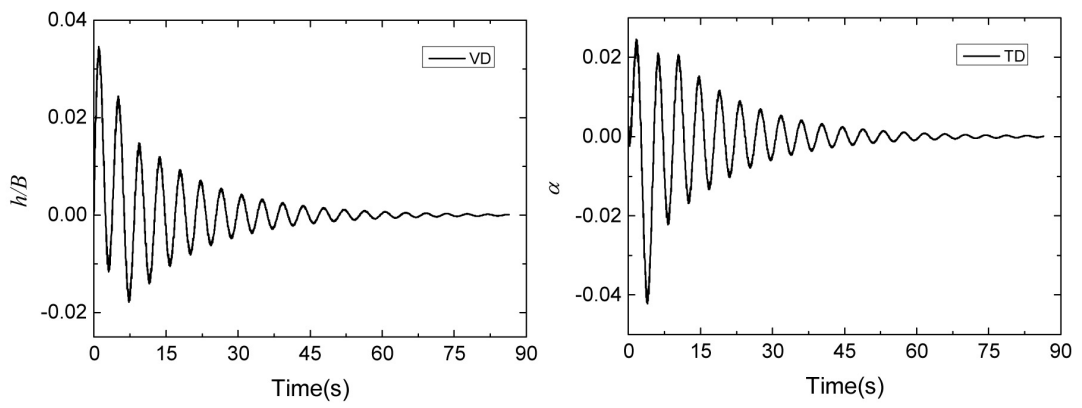

Figure 4. Time-history of system displacements under suboptimal control.

aerodynamic derivatives of the deck-flap system with only a few wind tunnel tests. But its accuracy need to be checked.

The numerical simulation shows that the aerodynamic flaps can suppress flutter on desired wind speed. Although feedback gain from the optimal control effectively stabilize the system in high wind speed, its state vector is not that easy to track. The suboptimal control can greatly suppress the vibration and relies on a few state vector parameters.

\section{References}

[1] Kobayashi, H. and Nagaoka, H. (1992) Active Control of Flutter of a Suspension Bridge. Journal of Wind Engineering and Industrial Aerodynamics, 41, 143-151. https://doi.org/10.1016/0167-6105(92)90402-V

[2] Hansen, H.I. and Thoft-Christensen, P. (1998) Active Vibration Control of Long Suspension Bridges Using Flaps. Proceedings of the 2nd World Conference on Structure Control, Wiley, Singapore.

[3] Guo, Z.W. and Ge, Y.J. (2012) A New State-Space Model for Self-Exited Forces and Flutter Automatic Analysis of Long Span Bridges. 7 th International Cooloquium on Bluff Body Aerodynamics and Applications (BBAA7), Shanghai, China.

[4] Theodorsen, T. (1935) General Theory of Aerodynamic Instability and the Mechanism of Flutter. Aerodynamic Flutter, American Institute of Aeronautics and Astronautics, No. 496.

[5] Dowell, E.H., Clark, R., Curtiss Jr., H.C., et al. (2004) A Modern Course in Aeroelasticity. 4th Edition, Kluwer Academic Publishers.

[6] Wilde, K. and Fujino, Y. (1998) Aerodynamic Control of Bridge Deck Flutter by Active Surfaces. Journal of Engineering Mechanics, 124, 718-727. https://doi.org/10.1061/(ASCE)0733-9399(1998)124:7(718)

[7] Librescu, L. and Marzocca, P. (2005) Advances in the Linear/Nonlinear Control of Aeroelastic Structural Systems. Atca Mechanica, 178, 147-186. https://doi.org/10.1007/s00707-005-0222-6 